\title{
Operational indistinguishably of varying speed of light theories
}

\author{
Nosratollah Jafari $^{1}$ and Ahmad Shariati ${ }^{2}$ \\ 1 Institute for Advanced Studies in Basic Sciences, \\ P.O. Box 159, Zanjan 45195, Iran \\ njafary@iasbs.ac.ir \\ 2 Department of Physics, Alzahra University, \\ Tehran 19938-91167, Iran. \\ shariati@mailaps.org
}

\begin{abstract}
The varying speed of light theories have been recently proposed to solve the standard model problems and anomalies in the ultra high energy cosmic rays. These theories try to formulate a new relativity with no assumptions about the constancy of the light speed. In this regard, we study two theories and want to show that these theories are not the new theories of relativity, but only re-descriptions of Einstein's special relativity.
\end{abstract}

PACS numbers: 03.30.+p, 11.30.Cp

\section{Introduction}

The varying speed of light theories has taken much attraction recently [1]. These theories have been proposed to solve some problems in the standard model cosmology, viz. the horizon and the flatness problems, and the anomalies in ultra high energy cosmic rays and $\mathrm{TeV}$ photons. The idea is that, may be Einstein's special relativity needs some modifications in very high energies or very large scales.

Einstein's special relativity is based on the following two principles:

1. The relativity principle: The physical laws are the same in all inertial frames.

2. The constancy of the speed of light: The speed of light is independent of the speed of observer and source.

These two principles, combined with some assumptions about the homogeneity of space and time, and isotropy of space, lead to Lorentz invariance.
One may ask the following question: "What if one relaxes Einstein's second postulate?" That is, what if one assumes that the speed of light, being finite, need not to be constant. There are some arguments, showing that if one assumes that the speed of light is finite, there is no way out of its being a universal constant [2] pp. 1-9]. In other words, there is no way out of the Lorentz group being the local symmetry group of our spacetime.

Now, there are some efforts to construct theories, alternative to the special relativity, in which the speed of light is not a constant. These are called "varying speed of light" theories (VLS). The Fock-Lorentz (FL) 3] and Magueijo-Smolin (MS) 4] transformations are two important examples of such theories. This article is a set of comments about these theories.

The main point is that these theories are basically re-description of Einstein's special relativity in noncartesian coordinates coordinates. This result is in agreement with those found by Ahluwalia-Khalilova [5] 6] and Grumiller et.al [].

\section{Fock-Lorentz Transforma- tions}

These transformations were obtained firstly by Fock [3] with no assumption about the constancy of light speed. In Fock's argument the most general transformations between two systems that are in uniform motion with respect to each other are linearfractional transformations. Recently, the same transformations are re-derived by Stepanov 8, 9] and Manida 10.

The FL transformations between systems $S$ and $S^{\prime}$ that are in relative motion with constant speed 
$v$ (along the common $x$-axis) are:

$$
\begin{aligned}
t^{\prime} & =\frac{\gamma\left(t-\frac{v}{c^{2}} x\right)}{1+\lambda v \gamma x-\lambda c^{2}(\gamma-1) t} \\
x^{\prime} & =\frac{\gamma(x-v t)}{1+\lambda v \gamma x-\lambda c^{2}(\gamma-1) t} \\
y^{\prime} & =\frac{y}{1+\lambda v \gamma x-\lambda c^{2}(\gamma-1) t} \\
z^{\prime} & =\frac{z}{1+\lambda v \gamma x-\lambda c^{2}(\gamma-1) t}
\end{aligned}
$$

where $\gamma=\left(1-\frac{v^{2}}{c^{2}}\right)^{-\frac{1}{2}}, c=299,792,458 \mathrm{~ms}^{-1}$, and $\lambda$ is a quantity with dimensions $L^{-2} T$, related to the Hubble's constant through $H \propto \lambda c^{2}$. If we assume $H \simeq 10^{2} \mathrm{~km} \mathrm{sec}^{-1} \mathrm{Mpc}^{-1}$, we have $\lambda \sim 10^{-35} \mathrm{~m}^{-2} \mathrm{~s}$, which shows that for all conceivable lengths and velocities, the denominators are very close to 1 . Therefore, these transformations are very close to the usual Lorentz transformations.

The FL transformations form a group, which is isomorphic to the Lorentz group. In fact, the FL transformations are a non-linear representation of the Lorentz group.

The most important difference between FL and usual Lorentz transformations is the variability of light speed. The speed that left invariant by the FL transformations instead of $c$ is

$$
C(t, \mathbf{x})=\left|\frac{\mathbf{c}+\lambda c^{2} \mathbf{x}}{1+\lambda c^{2} t}\right|
$$

here $\mathbf{c}=c \hat{n}$, where $\hat{n}$ is a unit vector, and $c$ is the constant $299,792,458 \mathrm{~ms}^{-1}$; and $C(t, \mathbf{x})$ is a function of space and time. $C$ is a decreasing function of time 8].

It is easily seen from the FL transformations that these transformations left invariant the expression

$$
\frac{c^{2} t^{2}-\mathbf{x} \cdot \mathbf{x}}{\left(1+\lambda c^{2} t\right)^{2}}
$$

that is

$$
\frac{c^{2} t^{2}-\mathbf{x} \cdot \mathbf{x}}{\left(1+\lambda c^{2} t\right)^{2}}=\frac{c^{2} t^{\prime 2}-\mathbf{x}^{\prime} \cdot \mathbf{x}^{\prime}}{\left(1+\lambda c^{2} t^{\prime}\right)^{2}} .
$$

Now, it is well known 8 that the FL spacetime is a Lorentzian spacetime with the following metric:

$$
\begin{aligned}
d s^{2}=\frac{1-\lambda^{2} c^{2} \mathbf{x} \cdot \mathbf{x}}{\left(1+\lambda c^{2} t\right)^{4}} c^{2} d t^{2} & +\frac{2 \lambda c^{2} \mathbf{x} \cdot d \mathbf{x} d t}{\left(1+\lambda c^{2} t\right)^{3}} \\
& -\frac{d \mathbf{x} \cdot d \mathbf{x}}{\left(1+\lambda c^{2} t\right)^{2}}
\end{aligned}
$$

Although this is a rather complicated metric, it can be shown that its Riemann tensor vanishes identically. Therefore, this spacetime is locally isometric

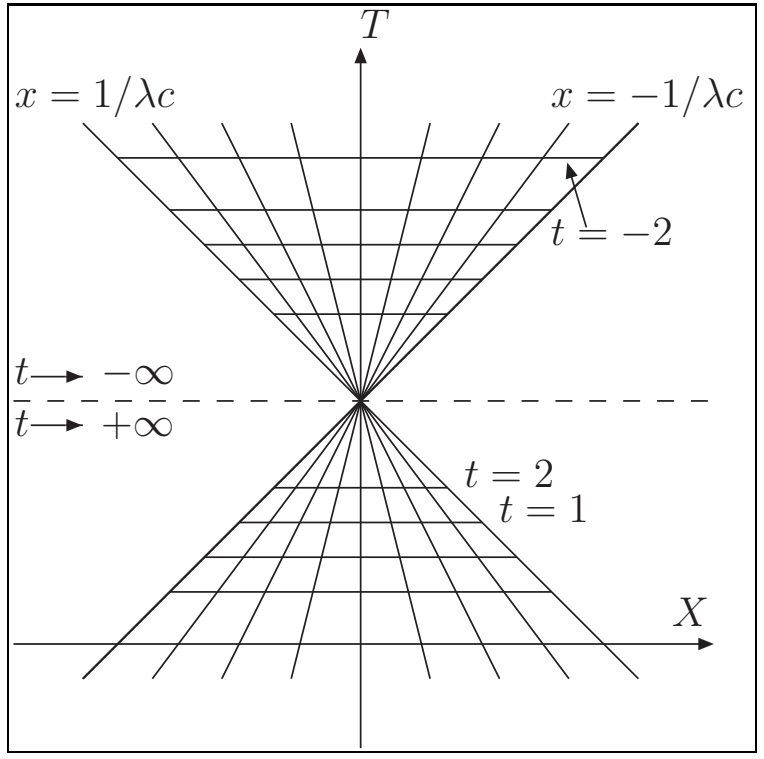

Figure 1: FL spacetime diagram in terms of $(T, X)$ coordinates. FL coordinates $t$ and $x$ is drawn as horizontal and radial lines.

to a part of the Minkowski spacetime. In fact, in the new coordinates

$$
\begin{aligned}
& T=\frac{t}{1+\lambda c^{2} t}, \\
& \mathbf{X}=\frac{\mathbf{x}}{1+\lambda c^{2} t}
\end{aligned}
$$

the metric reads

$$
d s^{2}=c^{2} d T^{2}-d X^{2}-d Y^{2}-d Z^{2} .
$$

Let's investigate the meaning of this transformation. Note that $T$ is the temporal coordinate, and $\mathbf{X}=(X, Y, Z)$ are cartesian coordinates, in an inertial frame. Coordinates $t$ and $\mathbf{x}:=(x, y, z)$ are being defined through these relations. Inverting this transformation, we get $t=T /\left(1-\lambda c^{2} T\right)$, $\mathbf{x}=\mathbf{X} /\left(1-\lambda c^{2} T\right)$. It is obvious that these transformations are not well defined on the hyperplane $T=1 /\left(\lambda c^{2}\right)$. This hyperplane is illustrated as a dashed line in fig 1 . From $T=t /\left(1+\lambda c^{2} t\right)$, or its equivalent $t=T /\left(1-\lambda c^{2} T\right)$, we know that the sections $T=$ constant, are the same as the sections $t=$ constant; only the labelling is different. Note that the section $T=1 /\left(\lambda c^{2}\right)$, is a hyperplane of discontinuity in $t$. Now from $\mathbf{x}=$ $\mathbf{X} /\left(1-\lambda c^{2} T\right)$, we see that the lines $\mathbf{x}=\left(x_{0}, y_{0}, z_{0}\right)$ are the lines $\mathbf{X}=\mathbf{x}-\lambda c^{2} \mathbf{x} T$. This means that, as far as $\lambda c^{2}|\mathbf{x}|<c$, one can interpret the line $\mathbf{x}=\left(x_{0}, y_{0}, z_{0}\right)$ as the worldline of a massive particle, or an observer. In other words, the set of lines 


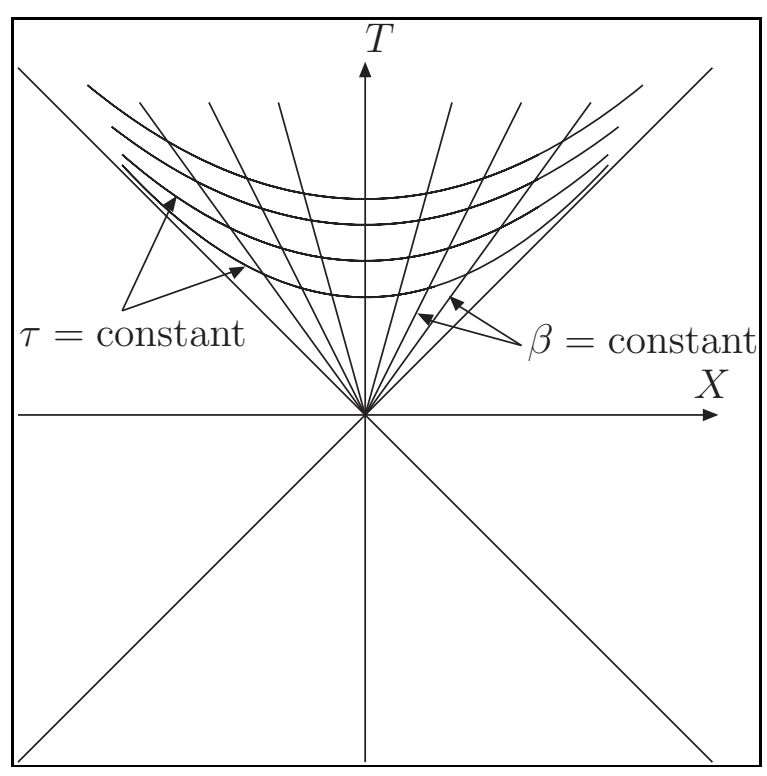

Figure 2: The Milne spacetime diagram in terms of $(T, X)$ coordinates.

$-\frac{1}{\lambda c}<|\mathbf{x}|<\frac{1}{\lambda c}$, which lie inside the lightcone with vertex $\left(T=1 /\left(\lambda c^{2}\right), X=0, Y=0, Z=0\right)$, can be interpreted as the worldlines of point observers. Therefore, inside the lightcone indicated in Fig. 1, one can interpret $\mathbf{x}$ and $t$ as spacial and temporal coordinates.

In terms of these new time and space coordinates, the FL transformations (14) reads

$$
\begin{aligned}
T^{\prime} & =\gamma\left(T-\frac{v}{c^{2}} X\right), \\
X^{\prime} & =\gamma(X-v T), \\
Y^{\prime} & =Y \\
Z^{\prime} & =Z
\end{aligned}
$$

This is the usual Lorentz transformations for $(T, X, Y, Z)$ coordinates. Thus, the FL transformations is solely a re-description of Einstein's special relativity in the unusual coordinates. Although this is quite well known, we think people do not pay enough attention to it.

It is useful to mention that the FL spacetime is the same as Milne spacetime. The Milne spacetime metric is $d s^{2}=d \tau^{2}-\tau^{2} d \beta^{2}$ and its spacetime diagram is shown in Fig.2. In Fig.2 the $\tau$-constant hyperbolae and the $\beta$-constant lines specify the Milne coordinates. The relation between the Milne coordinates $(\tau, \beta)$ and the Minkowskian coordinates $(T, X)$ is

$$
\tau=\sqrt{T^{2}-X^{2}}
$$

$$
\tanh \beta=\frac{X}{T}
$$

By means of these relations one can obtain the relation between the FL coordinates $(t, x)$ and the Milne coordinates $(\tau, \beta)$.

$$
\begin{aligned}
& \tau=\frac{\sqrt{t^{2}-x^{2}}}{1+\lambda c^{2} t}, \\
& \tanh \beta=\frac{x}{t} .
\end{aligned}
$$

All phenomena that are mentioned about the FL spacetime can also be seen in the Milne spacetime. Thus it seems that light speed is variable in this spacetime, too. Let us define the light speed as coordinate speed. From

$$
d s^{2}=d \tau^{2}-\tau^{2} d \beta^{2}=0,
$$

we get

$$
c_{\text {light }}:=\frac{d \beta}{d \tau}=\frac{1}{\tau} .
$$

With this definition, the light speed is large at initial moments and decrease with Milne time $\tau$. Therefore, the light speed that is variable in Stepanov and Manida viewpoint is the coordinate speed.

It is interesting to consider some particles in the origin of the Milne spacetime at $\tau=0$. These particles move away in the direction of the $\beta$-coordinates with passing time. This picture is the Milne enlarging universe picture and the rate of this enlargement depends on the $\lambda$ parameter, which is the Stepanov's 9] result about the Hubble's constant $H \propto \lambda c^{2}$.

\section{Magueijo-Smolin Transfor- mations}

Magueijo and Smolin generalize Fock's idea to the momentum space and found transformations that left invariant the Planck energy [4, 11]. Note that by the usual linear action of the Lorentz boosts on the four-momenta of particles, the Planck energy is not invariant. However, one can define a non-linear action of the Lorentz boosts on the four-momenta of particles, such that a specific energy scale, to be called the Planck scale, is invariant. When systems $S$ and $S^{\prime}$ are in relative motion with constant speed $v$ (along the common $x$-axis), these non-linear transformations read

$$
p_{0}^{\prime}=\frac{\gamma\left(p_{0}-\frac{v}{c} p_{x}\right)}{1+\ell(\gamma-1) p_{0}-\ell \gamma \frac{v}{c} p_{x}},
$$




$$
\begin{aligned}
p_{x}^{\prime} & =\frac{\gamma\left(p_{x}-\frac{v}{c} p_{0}\right)}{1+\ell(\gamma-1) p_{0}-\ell \gamma \frac{v}{c} p_{x}} \\
p_{y}^{\prime} & =\frac{p_{y}}{1+\ell(\gamma-1) p_{0}-\ell \gamma \frac{v}{c} p_{x}} \\
p_{z}^{\prime} & =\frac{p_{z}}{1+\ell(\gamma-1) p_{0}-\ell \gamma \frac{v}{c} p_{x}}
\end{aligned}
$$

where $\ell$ is a quantity with dimensions $M^{-1} L^{-1} T$, related to the Planck length $l_{p}$ through $\ell \propto l_{p} / \hbar$. (From now on we put $\hbar=1$, so that $\ell$ is the Planck length.)

These transformations are also, a non-linear representation of the Lorentz group in the momentum space, and depend on the ratio of the particle energy to Planck energy. If the particle energy is very small compared to the Planck energy, then these transformations become the usual Lorentz transformations. But looking at these transformations, we can see that changing 4 -momentum $p_{\mu}$ to

$$
P_{\mu}=\frac{p_{\mu}}{1-\ell p_{0}},
$$

then MS transformations become

$$
\begin{aligned}
P_{0}^{\prime} & =\gamma\left(P_{0}-\frac{v}{c} P_{x}\right), \\
P_{x}^{\prime} & =\gamma\left(P_{x}-\frac{v}{c} P_{0}\right), \\
P_{y}^{\prime} & =P_{y}, \\
P_{z}^{\prime} & =P_{z}
\end{aligned}
$$

These are, the same usual Lorentz transformations for momentum space. Therefore, the MS transformations, like the FL ones, are only re-description of the usual Lorentz transformations in the unusual coordinates. Also, it is easily seen that MS momentum space is the interior of the light-cone in the Minkowski momentum space. The MS momentum space diagram is a figure like Fig.1. Also, as in the FL case all MS results can be obtained from Einstein's special relativity by using the coordinate transformations (26). For example, $\|p\|^{2}$ invariant in the MS formalism can be viewed as

$$
\|p\|^{2}=\frac{\eta_{\mu \nu} p_{\mu} p_{\nu}}{\left(1-\ell p_{0}\right)^{2}}=\eta_{\mu \nu} P_{\mu} P_{\nu}=\|P\|^{2},
$$

where $\eta_{\mu \nu}=\operatorname{diag}(-1,1,1,1)$ is the Minkowski metric.

After giving the momentum transformations 22 25) by Magueijo 4, some people like Mignemi [12. and Magueijo himself [13] tried to find corresponding transformations in spacetime. Although, they approached the problem in different ways, they obtained the same result. Their transformations between two observers that are in relative motion with constant speed $v$ in two dimensions are

$$
\begin{gathered}
t^{\prime}=\Delta(t \cosh \xi-x \sinh \xi), \\
x^{\prime}=\Delta(-t \sinh \xi+x \cosh \xi),
\end{gathered}
$$

where

$$
\Delta=1+p_{0} \ell(\cosh \xi-1)+p_{1} \ell \sinh \xi,
$$

$\xi$ is the rapidity parameter and $\ell$ is the Planck length. The problem with these transformations is their energy and momentum dependence: Spacetime is a set of events to which we usually do not assign energy and momentum. Events occur in definite positions and times and differ from particles that have definite energies and momenta. Thus, the energy and momentum dependency of the spacetime transformations picture is hard to be meaningful.

However, the reverse procedure has no difficulty. For example, we can look for the momentum transformations corresponding to the FL spacetime transformations. In this case we have for two dimensions

$$
\begin{array}{ll}
p_{0}^{\prime}=\quad & \tilde{\Delta}[\cosh \xi+\lambda t(\cosh \xi-1)] p_{0} \\
+ & \tilde{\Delta}[\sinh \xi+\lambda x(\cosh \xi-1)] p_{1}, \\
p_{1}^{\prime}=\quad & \tilde{\Delta}[\sinh \xi+\lambda t \sinh \xi] p_{0} \\
+ & \tilde{\Delta}[\cosh \xi+\lambda x \sinh \xi)] p_{1},
\end{array}
$$

where

$$
\tilde{\Delta}=1+\lambda x \sinh \xi-\lambda(\cosh \xi-1) t,
$$

and $\lambda$ is a small quantity related to Hubble's constant. In these transformations, momenta depend on the position and time. This is not absurd, because a classical particle, at each instant, is at a definite position.

\section{Conclusion}

It is outlined that the MS and FL transformations are only re-descriptions of Einstein's special relativity in one portion of the Minkowski spacetime. In other words, Einstein's special relativity still remains valid, and these transformations are only descriptions of the special relativity in the language of non-cartesian coordinates that lead to the apparent variability of the speed of light, and apparent violation of the Lorentz invariance. 


\section{Acknowledgements}

We should thanks our colleagues M. Khorrami and A.H. Fatollahi at IASBS for their kind discussions. We are also grateful to D.V. Ahluwalia-Khalilova, A.F. Ranada, D.V. Vassilevich, R. Lehnert and D. Grumiller for helping us put our work in perspective.

\section{References}

[1] J. Magueijo, "New varying speed of light theories", Rept. Prog. Phys. 66 (2003) 2025, arXiv: astro-ph/0305457

[2] Roman U. Sexl and H. K. Urbantke: Relativity, Groups, Particles-Special Relativity and Relativistic Symmetry in Field and Particle Physics; Springer, Wien, 2001.

[3] V. A. Fock, "The theory of spacetime and gravitation" Pregmon Press, 1964.

[4] J. Magueijo and L. Smolin, "Lorentz invariance with an invariant energy scale", Phys. Rev. Lett.88 (2002) 190403.

[5] D. V. Ahluwalia-Khalilova, "Operational indistinguishability of double special relativities from special relativity", arXiv: gr-qc/0212128

[6] D. V. Ahluwalia-Khalilova, "Fermions, bosons, and locality in special relativity with two invariant scales", arXiv: gr-qc/0207004

[7] D. Grumiller, W. Kummer, D. V. Vassilevich "A note on the triviality of $\kappa$-deformations of gravity", Ukr. J. Phys. 48 (2003) 329, arXiv: hep-th/0301061

[8] S. S. Stepanov, "Fundamental physical constants and the principle of parametric incompleteness", arXiv: physics/9909009.

[9] S. S. Stepanov, "Time-space varying speed of light and the Hubble law in a static universe", Phys. Rev. D 62 (2000) 023507.

[10] S. N. Manida, "Fock-Lorentz transformations and time-varying speed of light", arXiv: gr-qc/9905046

[11] J. Magueijo and L. Smolin, "Generalized Lorentz invariance with an invariant energy scale", Phys. Rev. D 67 (2003) 044017.
[12] S. Mignemi, "Transformations of coordinates and Hamiltonian formalism in deformed special relativity", Phys. Rev. D 68 (2003) 065029 .

[13] D. Kimberly, J. Magueijo and J. Medeiros, "Non-linear relativity in position space", arXive: gr-qc/0303067. 